\title{
Aplicação da técnica de CAMQL na retificação cilíndrica externa do aço AISI 4340 utilizando rebolo de CBN
}

\author{
Application of CAMQL technique on \\ external cylindrical grinding of AISI \\ 4340 steel with CBN grinding wheel
}

Kamira Miksza Fragoso ${ }^{1}$,Alan Polato Francelin ${ }^{1}$, José Claudio Lopes ${ }^{1}$, Luiz Eduardo de Angelo Sanchez ${ }^{1}$, Hamilton José de Melo ${ }^{1}$, Alexandre Araujo Lodi ${ }^{2}$, Paulo Roberto Aguiar ${ }^{1}$, Eduardo Carlos Bianchi ${ }^{1}$

\footnotetext{
${ }^{1}$ Universidade Estadual Paulista - UNESP, Faculdade de Engenharia de Bauru. Av. Luiz Edmundo Carrijo Coube, 14-01, Bauru, São Paulo, Brasil.

e-mail: kah.miksza@gmail.com, alan.polato@gmail.com, jclaudio.lopes@hotmail.com, sanchez@feb.unesp.br, hamilton@feb.unesp.br, aguiarpr@feb.unesp.br, bianchi@feb.unesp.br

${ }^{2}$ Emuge Franken. Rod. Das Estâncias, km90 + 692 mts, Itatiba, São Paulo, Brasil.

e-mail: alexandre.lodi@emuge-franken.com.br
}

\section{RESUMO}

O processo de retificação possui como características o bom acabamento superficial e alta precisão dimensional e geométrica. Assim, peças usinadas por este processo possuem um alto valor agregado por se tratar de um processo de acabamento que é comumente realizado nas últimas etapas da fabricação. Um fator limitador do processo de retificação é a quantidade de calor gerada, a qual é minimizada pela aplicação de fluidos de corte. Os fluidos utilizados, na maior parte dos casos são poluentes e contém substâncias que são nocivas à saúde e ao meio ambiente, além do mesmo possuir um alto custo de compra, manutenção e descarte. Visando reduzir a quantidade de fluido utilizada durante o processo de retificação, o presente trabalho foi desenvolvido. O seu principal objetivo é melhorar a técnica da mínima quantidade de lubrificação através da aplicação combinada com ar comprimido a baixa temperatura. Esta aplicação visa aumentar o efeito de refrigeração e assim minimizar a geração de calor na região de corte. Para avaliar a eficácia da técnica foram analisados os parâmetros de rugosidade, circularidade, desgaste do rebolo e potência consumida durante a usinagem. Estes valores foram medidos para três métodos distintos de aplicação de fluidos de corte durante o processo de retificação, a fim de compará-los. A técnica de lubri-refrigeração convencional obteve melhores resultados para os parâmetros analisados, ficando a técnica de CAMQL (mínima quantidade de fluido combinado com a aplicação de ar comprimido resfriado) com resultados intermediários e a técnica de MQL (mínima quantidade de lubrificação) com os piores resultados obtidos em todos os parâmetros. A técnica proposta de redução de quantidade de fluido utilizada atingiu, em geral, resultados positivos, provando a viabilidade da mesma para a retificação do aço AISI 4340 utilizando rebolo de CBN, o Nitreto Cúbico de Boro, um superabrasivo com capacidade de corte inferior apenas ao diamante.

Palavras-chave: Retificação, Rebolo de CBN, CAMQL, ar frio, aço AISI 4340.

\section{ABSTRACT}

The grinding process provides us with good surface finish and good dimensional and geometric precision. Also, ground pieces have a high added value due to this process being a finishing one and is commonly carried out in the last stages of manufacture. A limiting factor for grinding is the great amount of heat generated, which is better removed from the cutting zone by the application of cutting fluids. The fluid used, in most of cases, are pollutants and contain substances that are harmful both to workers and environment, besides having a high cost of purchase, maintenance and disposal. In order to reduce the amount of fluid used during grinding, the present work was developed. Its main objective is to improve the technique of minimum quantity of lubrication (MQL) by combining it with the application of compressed cold air. This aims to increase 
the cooling effect and yet minimize the heat concentration in the cutting zone. To evaluate the effectiveness of the technique, the parameters of roughness, circularity, grinding wheel wear and power consumption during machining were analyzed. These values were measured for three different methods of application of cutting fluids during grinding to compare. The conventional flood application obtained the best results for the parameters analyzed while CAMQL (cold air minimum quantity lubrication) presents intermediate results and MQL (minimum quantity lubrication) the worst results obtained in all analyzed parameters. The proposed technique of reducing the amount of fluid used has generally been positive, proving its feasibility for the grinding of AISI 4340 steel using CBN grinding wheel with CBN being Cubic Boron Nitride, a superabrasive material.

Keywords: Grinding, CBN grinding wheel, CAMQL, cold air, AISI 4340 steel.

\section{INTRODUÇÃO}

A utilização de fluido de corte demanda onerosos investimentos tanto para a sua aquisição como para manutenção [1]. Também é necessário que o mesmo seja descartado de forma adequada, pois o fluido de corte possui elementos nocivos em sua composição, como cloro, enxofre e fósforo [2]. Estes elementos, além de serem poluentes, contaminando tanto o solo quando águas superficiais e subterrâneas e ainda podem ser prejudiciais à saúde do operador [3]. O fluido de corte eventualmente possui detritos metálicos provenientes da usinagem e também pode favorecer a proliferação de bactérias. Esta combinação pode ocasionar problemas de pele, respiratórios e digestivos. Somado a isso tem-se atualmente leis ambientais mais rígidas, tanto para a proteção ambiental como para a proteção aos operadores [4]. Todos estes fatores contribuem com pesquisas voltadas para a redução ou completa eliminação do uso de fluidos na indústria de fabricação do futuro sem que haja perdas em qualidade ou produtividade.

A busca por alternativas para a lubri-refrigeração convencional no processo de retificação tem conduzido a opções que preservam tanto a saúde do operador como o meio ambiente, podendo também agregar valor ao processo e reduzir os custos de produção. Uma destas alternativas é a retificação a seco, onde a usinagem se realiza sem nenhum uso de fluido.

Um exemplo foi desenvolvido por DEBNATH et al. [5], onde realizou-se a retificação a seco com resfriamento prévio das peças em nitrogênio líquido. Isso possibilitou que a amostra estivesse em baixa temperatura durante o processo de usinagem. O estudo foi focado em processos que não eram críticos para queima, como por exemplo, materiais macios e de fácil usinagem. Os corpos de prova utilizados foram fabricados de aço AISI 1040 e foram mantidos em banho de nitrogênio durante $100 \mathrm{~s}$. Apesar de resultados positivos em termos de acabamento e qualidade superficial, a viabilidade da técnica deve ser testada para outras aplicações.

Uma opção para a redução no uso de fluidos de corte durante a retificação é a técnica de mínima quantidade de lubrificação (MQL). Esta técnica consiste em pulverizar uma pequena quantidade de óleo em um jato de ar comprimido, que será direcionado para a zona de corte. Esta técnica combina a funcionalidade da refrigeração com um consumo reduzido de fluido. As limitações da retificação a seco podem ser superadas com esta técnica. [3];[8]

Visando melhorar o parâmetro de refrigeração que é deficiente na técnica de MQL algumas pesquisas com diferentes soluções foram realizadas. Como por exemplo BELENTANI et al. [3] que combinou a técnica de MQL com um fluido de base água a fim de melhorar a capacidade de arrefecimento do processo. Utilizou-se três diferentes proporções de água (1:1, 1:3 e 1:5) na retificação do aço AISI 4340. Conclui-se que o aumento da quantidade de água no óleo melhora o acabamento superficial, melhora as variáveis de forma, diminui o desgaste e as forças de usinagem. Isso acontece pois há menos empastamento do rebolo devido à baixa viscosidade do fluido misturado com água.

Outra alternativa para o problema do arrefecimento da técnica de MQL é a aplicação combinada da mesma com fluido gasoso resfriado, como por exemplo $\mathrm{CO}_{2}$ em baixa temperatura, conforme desenvolvido por BEN FREDJ et al. [10] e por MORGAN et al. [11]. Ambos tratam da aplicação combinada de MQL e de $\mathrm{CO}_{2}$ em baixa temperatura em bocais separados, a fim de que se possa solucionar o problema de falta de refrigeração acarretado pela técnica de mínima quantidade de lubrificação. BEN FREDJ et al. [10] verificou uma redução em grande escala do desgaste do rebolo, aumentado assim sua vida, com um acabamento ligeiramente melhor para o sistema estudado, porém as forças tenderam a ser levemente maiores com o sistema proposto. Já segundo MORGAN et al. [11], a lubri-refrigeração convencional apresentou melhores resultados, porém tendo em vista que o objetivo era a redução do uso de fluidos, a técnica proposta apresentou melhores resultados do que a aplicação de MQL convencional. 
A última técnica citada é a aplicação combinada de MQL com ar comprimido resfriado (CAMQL). Para tanto é utilizado um único bocal desenvolvido para este fim. SABERI et al. [12] comparou a técnica supracitada com a lubri-refrigeração convencional e com a retificação a seco. Os testes foram realizados para a retificação plana do aço AISI CK 45 utilizando rebolo de óxido de alumínio. Concluiu-se que a pressão do ar é um fator mais importante que a variação de temperatura, uma pressão maior com uma temperatura alta acarreta em melhores resultados quando comparado a uma pressão menor e baixa temperatura. Concluiu-se também que o método testado não é melhor que a retificação utilizando o método de lubri-refrigeração convencional, porém ele atinge a eficiência para a redução da quantidade de fluido usada, já que ela é melhor que a retificação a seco.

A revisão da literatura mostra que as mais diversas técnicas têm sido estudadas para buscar a solução para a redução da quantidade de fluido de corte utilizada no processo de retificação, de modo competitivo com a técnica de lubri-refrigeração convencional.

Este estudo foi desenvolvido para a técnica combinada da mínima quantidade de lubrificação com ar comprimido resfriado, ou simplesmente CAMQL, a fim de que seja possível a aplicação desta técnica de forma eficaz, na retificação do aço AISI 4340 temperado e revenido utilizando rebolo de CBN.

\section{MATERIAIS E MÉTODOS}

O equipamento utilizado para a realização do presente estudo foi a uma retificadora cilíndrica de mergulho modelo RUAP 515H da empresa Sulmecânica com comando numérico computadorizado CNC Fagor. A escolha dos parâmetros utilizados para os testes foi realizada com base em estudos preliminares, e se encontram na tabela 01 .

Tabela 1: Parâmetros utilizados nos testes de retificação

\begin{tabular}{|c|c|}
\hline RETIFICADORA & CNC RUAP 515 H SULMECÂNICA \\
\hline SISTEMA DE MQL & ACCU-LUBE 79053D \\
\hline TUBO DE VÓRTICE & EMUGUE-FRANK \\
\hline TIPO DE RETIFICAÇÃO & CILÍNDRICA DE MERGULHO \\
\hline REBOLO & CBN (SNB151Q12VR2) \\
\hline DIMENSÕES DA PEÇA & $54 \mathrm{~mm} \times 30 \mathrm{~mm} \times 4 \mathrm{~mm}$ \\
\hline VELOCIDADE PERIFÉRICA DO REBOLO & $33 \mathrm{~m} / \mathrm{s}$ \\
\hline VELOCIDADE DE AVANÇO & $0,25 \mathrm{~mm} / \mathrm{min} ; 0,50 \mathrm{~mm} / \mathrm{min} ; 0,75 \mathrm{~mm} / \mathrm{min}$ \\
\hline TEMPO DE SPARK-OUT & $7,5 \mathrm{~S}$ \\
\hline NÚMERO DE PASSES & 70 \\
\hline AVANÇO POR PASSE & $100 \mu \mathrm{m}$ \\
\hline VOLUME DE MATERIAL REMOVIDO & $2221 \mathrm{~mm}^{3}$ \\
\hline CONDIÇÕES DE LUBRIFICAÇÃO & CONVENCIONAL, MQL, CAMQL \\
\hline FLUIDO CONVENCIONAL & QUIMATIC ME-1 COM CONCENTRAÇÃO DE 3\% \\
\hline FLUIDO MQL & LB 1100 \\
\hline FLUIDO CAMQL & LB 1100 \\
\hline VAZÃO DE FLUIDO CONVENCIONAL & $550 \mathrm{~L} / \mathrm{h}$ \\
\hline VAZÃO DE FLUIDO MQL/CAMQL & $120 \mathrm{~mL} / \mathrm{h}$ \\
\hline PRESSÃO MQL/CAMQL & 6 bar \\
\hline TEMPERATURA CAMQL & $0^{\circ} \mathrm{C}$ \\
\hline DRESSADOR & CONGLOMERADO \\
\hline NÚMERO DE PASSES DE DRESSAGEM & 40 \\
\hline PROFUNDIDADE DE DRESSAGEM & $2 \mu \mathrm{m}$ \\
\hline VELOCIDADE DE DRESSAGEM & $600 \mathrm{~mm} / \mathrm{min}$ \\
\hline
\end{tabular}


Os corpos retificados possuem formato anelado, conforme a figura 01 e foram fabricadas de aço AISI 4340 temperado e revenido, com dureza de $54 \pm 2 \mathrm{HRc}$, cuja composição química se encontra na tabela 02 . O mesmo possui $54 \mathrm{~mm}$ de diâmetro externo, $30 \mathrm{~mm}$ de diâmetro interno e $4 \mathrm{~mm}$ de espessura. O rebolo usado para os testes foi de CBN com ligante vitrificado do tipo 14A1, de liga com dureza R, granulometria de 100 mesh, que possui $350 \mathrm{~mm}$ de diâmetro externo, $127 \mathrm{~mm}$ de diâmetro interno, $15 \mathrm{~mm}$ de largura e $5 \mathrm{~mm}$ de camada, o rebolo de código SNB151Q12VR2 doado pela Nikon Ferramentas de Corte Ltda - Saint-Gobain.

Tabela 2: Composição química do corpo de prova, fonte: [13]

\begin{tabular}{|l|l|l|l|l|l|}
\hline $\mathrm{C}$ & $0,4 \%$ & $\mathrm{~S}$ & $0,011 \%$ & $\mathrm{Al}$ & $0,016 \%$ \\
\hline $\mathrm{Mn}$ & $0,77 \%$ & $\mathrm{Cr}$ & $0,81 \%$ & $\mathrm{Cu}$ & $0,18 \%$ \\
\hline $\mathrm{P}$ & $0,026 \%$ & $\mathrm{Ni}$ & $1,71 \%$ & $\mathrm{Co}$ & $0,04 \%$ \\
\hline $\mathrm{Si}$ & $0,30 \%$ & $\mathrm{Mo}$ & $0,22 \%$ & $\mathrm{Fe}$ & BALANÇO \\
\hline
\end{tabular}

A aplicação de fluído de corte pelo método convencional foi realizada utilizando o sistema aplicador da própria máquina ferramenta, composto de reservatório, bomba e bicos aplicadores. O fluido utilizado foi o óleo solúvel semi-sintético QUIMATIC ME-1, com concentração de 3\% em água.

A aplicação da técnica de mínima quantidade de lubrificação foi realizada utilizando o aplicador de MQL Accu-Lube 79053D e um bocal desenvolvido para este fim. Já a técnica de mínima quantidade de lubrificação combinada com ar frio, denominada CAMQL foi aplicada utilizando o mesmo aplicador, porém com um bocal desenvolvido especificamente para a aplicação desta técnica e um tubo de vórtice para o resfriamento do ar comprimido fornecido pela Emugue-Frank, conforme a figura 01. A figura 02 ilustra a diferença de aplicação para as duas técnicas.

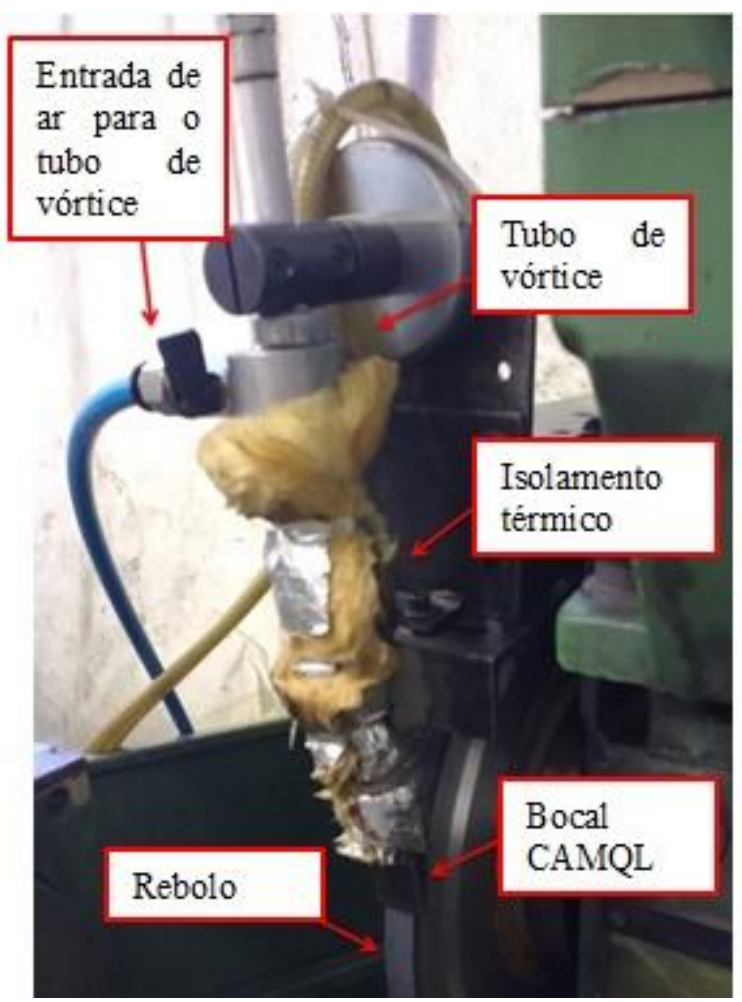

Figura 1: Detalhamento do esquema de aplicação de CAMQL. 
a) MQL

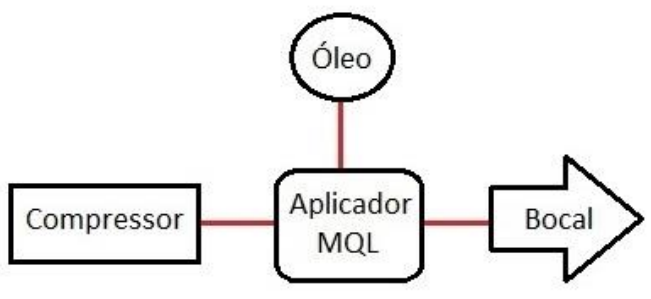

b) CAMQL

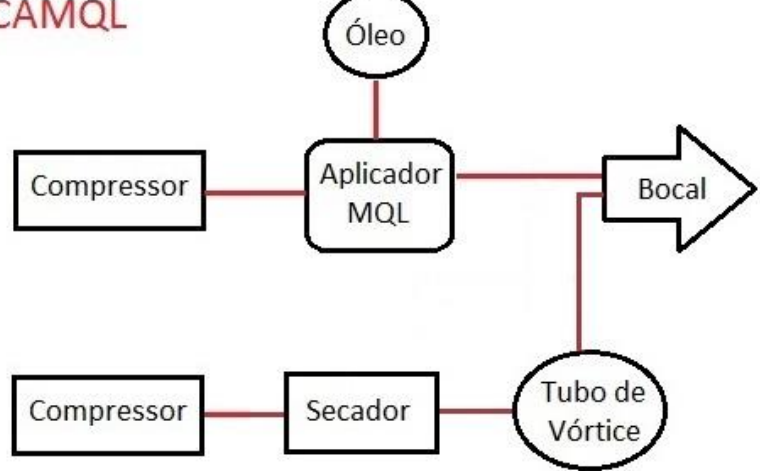

Figura 2: Diferença dos sistemas de aplicação: (a) MQL; (b) CAMQL.

Para as aplicações de MQL e CAMQL foi utilizado o fluido de corte Accu-Lube LB-1100 da empresa ITW Chemical Products Ltda.

Para cada condição definida foram realizados 3 ensaios, totalizando 27 amostras, conforme a figura 3.

Figura 3: Banco de ensaios
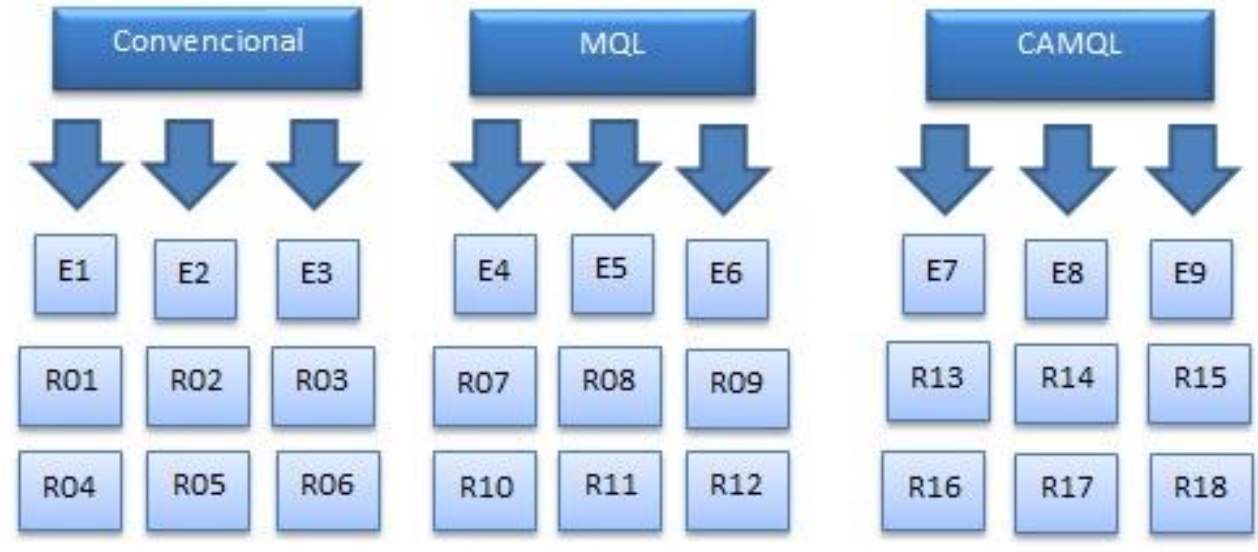

Os três primeiros ensaios realizados utilizaram o método de lubri-refrigeração convencional por inundação, com três diferentes velocidades de avanço, $0,25 \mathrm{~mm} / \mathrm{min}, 0,50 \mathrm{~mm} / \mathrm{min}$ e $0,75 \mathrm{~mm} / \mathrm{min}$. Em seguida foram realizados ensaios às três velocidades de avanço para as técnicas de mínima quantidade de lubrificação e para a técnica proposta de mínima quantidade de lubrificação com ar frio.

A velocidade de corte foi mantida constante em $33 \mathrm{~m} / \mathrm{s}$. A dressagem foi realizada após cada um dos ensaios utilizando um dressador conglomerado. A dressagem foi feita para garantir a mesma condição do rebolo para cada um dos testes e impedir assim que erros se acumulem nos resultados obtidos. Ela foi realizada em 40 passes de profundidade de $2 \mu \mathrm{m}$ cada, totalizando uma remoção de $80 \mu \mathrm{m}$ da superfície de corte do rebolo.

As variáveis de saída para a análise do processo foram a rugosidade da superfície usinada, a circularidade, o desgaste diametral do rebolo e a potência de corte consumida durante o processo. Estas variáveis foram escolhidas a fim de determinar a eficácia do processo.

\subsection{Rugosidade (Ra)}

A rugosidade média aritmética eu Ra foi escolhida como um dos parâmetros para a análise da qualidade superficial das amostras. A quantificação deste parâmetro foi realizada com um rugosímetro Surtronic 3+ da marca Taylor Hobson. Foram realizadas cinco medições em cada uma das amostras, tomadas com uma distância de aproximadamente $70^{\circ}$ umas das outras. Utilizou-se um cut-off de 0,25 mm e um comprimento de 
amostragem de $1,25 \mathrm{~mm}$.

\subsection{Circularidade}

Os desvios de circularidade foram escolhidos para verificação da precisão da forma das peças usinadas. A medição desses desvios foi realizada utilizando um medidor de desvios de forma modelo Tayround $31 \mathrm{C}$ da marca Taylor Hobson.

A fim de verificar a repetibilidade das medidas, elas foram realizadas em três posições diferentes, com uma distância de $0,5 \mathrm{~mm}$ entre elas.

\subsection{Desgaste diametral do rebolo}

O desgaste diametral do rebolo foi escolhido como parâmetro para a verificação da eficácia do processo em termos de aproveitamento e vida do rebolo e foi realizado com a técnica de impressão de perfil. Esta consiste em desgastar um cilindro de aço de baixa dureza (AISI 1020), com um volume fixo de material removido a fim de que o desgaste do rebolo fique marcado no corpo de prova. O desnível impresso no cilindro é então medido com o auxílio do rugosímetro, utilizando e seu próprio apalpador. O perfil de desnível é então ilustrado conforme a figura 03 . Nela, a diferença "a" é a medida do desgaste do rebolo.

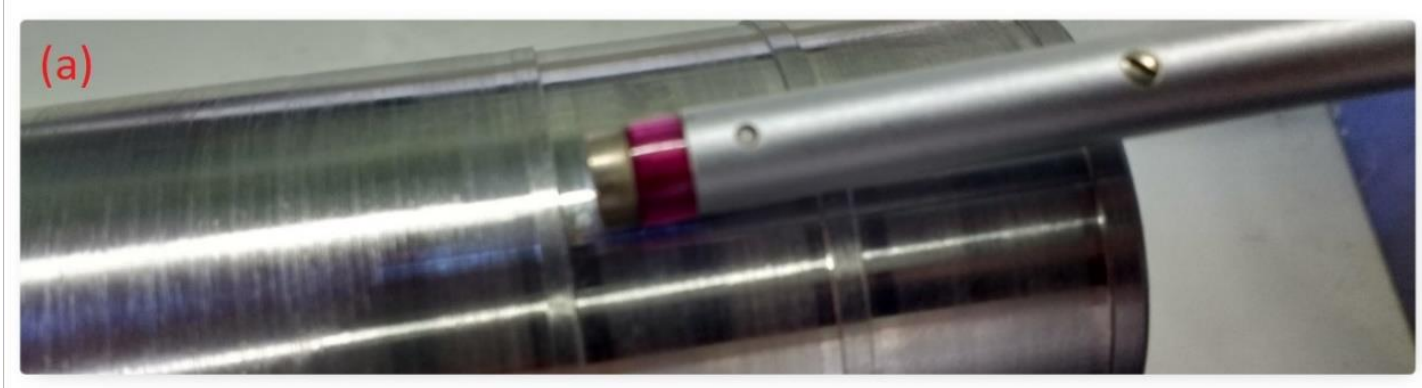

(b)

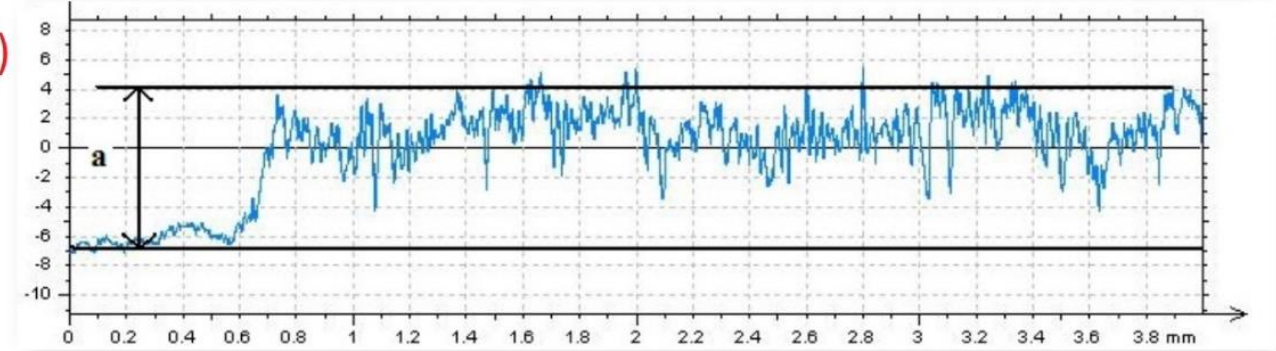

Figura 4: Perfil de desgaste diametral do rebolo marcado no tarugo de verificação do desgaste.

\subsection{Potência de corte}

Por fim tem-se o parâmetro selecionado para o monitoramento e avaliação do processo de retificação investigado no presente estudo. Assim, foi selecionada a potência consumida durante o processo de usinagem. Para tanto foi utilizado um sistema de aquisição da potência consumida pelo motor de acionamento da retificadora.

O processo de aquisição e tratamento de sinais foi realizado pelo software LabView 6.1®da empresa National Instruments.

Primeiramente os valores RMS de corrente elétrica e tensão provenientes do motor elétrico da retificadora CNC foram transformados através de um circuito eletrônico, chamado Curvopower, em sinais de tensão compatíveis com uma placa de aquisição de dados para que os mesmos pudessem então ser convertidos em valores de potência.

\section{RESULTADOS}

Este tópico apresenta os resultados obtidos conforme o procedimento descrito anteriormente. Todos os resultados obtidos encontram-se em função das velocidades de avanço utilizadas durante o experimento. Os resultados são expostos em termos da rugosidade média aritmética, circularidade, desgaste diametral do rebolo e 
potência de corte consumida durante o processo.

\subsection{Rugosidade}

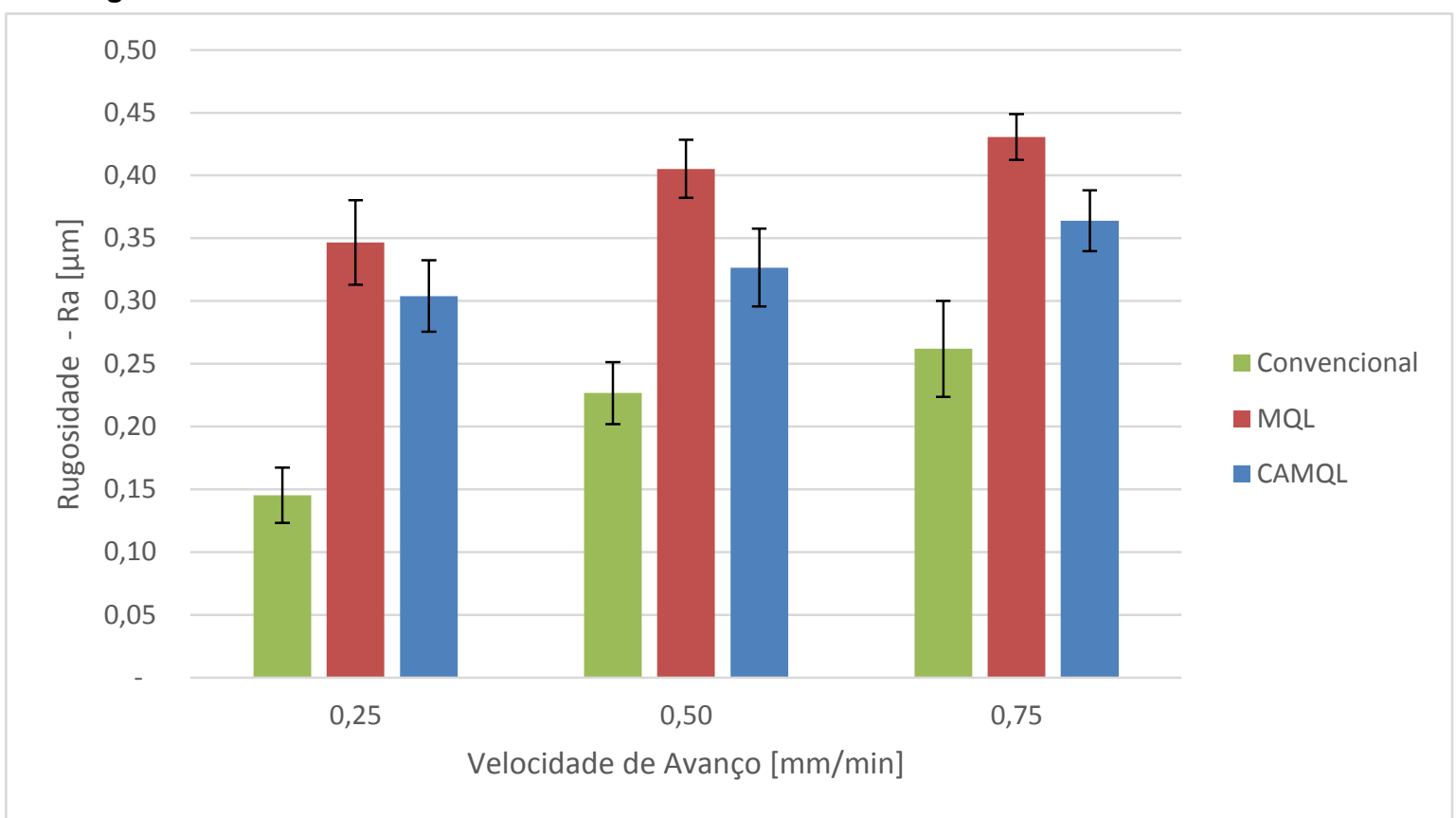

Figura 5: Dados obtidos de rugosidade ( $\mathrm{Ra})$ em função da velocidade de avanço.

Como é possível observar na figura 04, os resultados relativos à rugosidade seguiram a tendência de aumentar com o aumento da velocidade de avanço. Isso acontece, pois há um aumento da espessura de cavaco com o aumento da velocidade de avanço e consequentemente, a usinagem é considerada mais severa, e, portanto, há uma diminuição da qualidade superficial das peças.

A usinagem com a técnica de lubri-refrigeração convencional fornece os melhores resultados de rugosidade, com Ra de $0,15 \mu \mathrm{m}$ para a velocidade de avanço de $0,25 \mathrm{~mm} / \mathrm{min}$; Ra de $0,23 \mu \mathrm{m}$ para a velocidade de avanço de $0,50 \mathrm{~mm} / \mathrm{min}$ e Ra de $0,26 \mu \mathrm{m}$ para a velocidade de avanço de $0,75 \mathrm{~mm} / \mathrm{min}$. Isso acontece pois a lubri-refrigeração convencional tem a maior capacidade, entre os métodos testados, para remover cavacos da região de corte e isso faz com que a superfície do corpo de prova usinado não seja arranhada pelos mesmos e também há uma diminuição do atrito gerado, já que o processo com esse tipo de técnica fornece uma boa lubrificação na interface rebolo/peça, e, por fim, a presença de um grande volume de fluido permite que o rebolo se mantenha sem a formação de borra. O que leva ao melhor desempenho do processo, como pode ser observado na figura 05 .

A usinagem com a técnica de mínima quantidade de lubrificação ou MQL fornece os maiores resultados de rugosidade obtidos, com Ra de $0,35 \mu \mathrm{m}$ para a velocidade de avanço de $0,25 \mathrm{~mm} / \mathrm{min}$, Ra de $0,41 \mu \mathrm{m}$ para a velocidade de avanço de $0,50 \mathrm{~mm} / \mathrm{min}$ e Ra de $0,43 \mu \mathrm{m}$ para a velocidade de avanço de $0,75 \mathrm{~mm} / \mathrm{min}$. A técnica de MQL não promove refrigeração suficiente quando se compara com o método convencional, e este problema aumenta a quantidade de calor transferida para a peça durante a usinagem, e, em diversos estudos foi constatado que o aumento da quantidade de calor no processo reduz de forma significativa o acabamento das peças. A técnica de MQL promove a formação de uma borra, formada por óleo e cavaco na superfície do rebolo, o que acarreta também em um aumento da rugosidade. [2]

Por fim a técnica de mínima quantidade de lubrificação com ar comprimido resfriado (CAMQL) fornece os resultados intermediários para a rugosidade, com Ra de $0,30 \mu \mathrm{m}$ para a velocidade de avanço de 0,25 $\mathrm{mm} / \mathrm{min}$, Ra de $0,33 \mu \mathrm{m}$ para a velocidade de avanço de $0,50 \mathrm{~mm} / \mathrm{min}$ e Ra de $0,36 \mu \mathrm{m}$ para a velocidade de avanço de $0,75 \mathrm{~mm} / \mathrm{min}$. É possível notar que os resultados para a retificação na condição mais suave forneceram dados próximos para as técnicas de MQL e CAMQL, Ra de 0,35 $\mu \mathrm{m}$ e Ra de 0,30 $\mu \mathrm{m}$, respectivamente, isso acontece, pois como a usinagem acontece de forma mais branda há uma menor geração de calor e energia acumulada durante o processo e consequentemente isso acarreta em uma menor geração de borra e um acabamento bastante próximo para as duas técnicas de aplicação da mínima quantidade de refrigeração. Porém, para as outras duas condições estudadas os resultados se mantiveram com uma maior diferença uma 
vez que, como a técnica de CAMQL promove uma melhor refrigeração do processo há uma menor geração de calor e consequentemente tem-se menos energia acumulada na interface de corte. A técnica de CAMQL também ocasiona a formação de borra na superfície de corte do rebolo, portanto seus resultados ainda são maiores que aqueles obtidos pela técnica de lubri-refrigeração convencional.

\subsection{Circularidade}

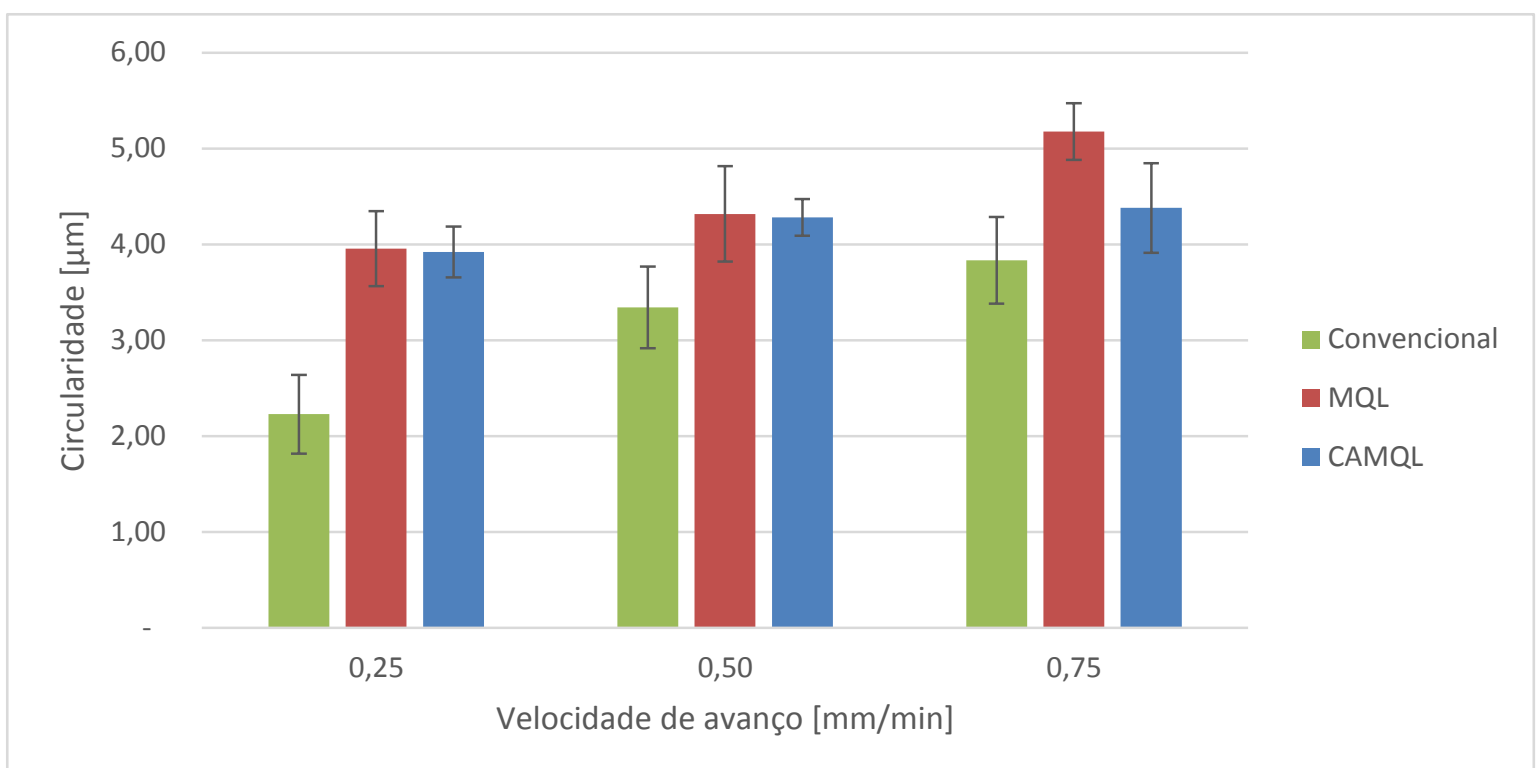

Figura 6: Desvio de circularidade de diferentes condições de lubri-refrigeração em função da velocidade de avanço.

É possível observar que os dados de circularidade apresentam a mesma tendência dos dados da rugosidade. Porém os resultados de MQL e CAMQL para as duas primeiras velocidades de avanço se mostram similares, isso se dá devido à formação de uma "borra" na superfície do rebolo causada pelo acumulo de cavaco e óleo lubrificante nos poros do mesmo, bem como à menor capacidade de remover calor que a técnica de MQL possui, seja ela em sua forma convencional ou utilizando ar comprimido em baixa temperatura, quando comparada à técnica de lubri-refrigeração convencional.

Para a técnica de MQL, os resultados de circularidade foram 3,96 $\mu \mathrm{m}, 4,32 \mu \mathrm{m}$ e $5,18 \mu \mathrm{m}$ para as velocidades de avanço de $0,25 \mathrm{~mm} / \mathrm{min} ; 0,50 \mathrm{~mm} / \mathrm{min}$ e $0,75 \mathrm{~mm} / \mathrm{min}$ respectivamente. Estes foram os resultados mais altos obtidos, pois como esta técnica não apresenta refrigeração suficiente para compensar a geração de calor e a energia acumulada durante a usinagem e isso acarreta em uma pior precisão dimensional.

Já para a técnica de CAMQL os resultados de circularidade foram 3,92 $\mu \mathrm{m}$ para a velocidade de avanço de $0,25 \mathrm{~mm} / \mathrm{min}, 4,28 \mu \mathrm{m}$ para a velocidade de avanço de $0,50 \mathrm{~mm} / \mathrm{min}$ e 4,38 $\mu \mathrm{m}$ para a velocidade de avanço de $0,75 \mathrm{~mm} / \mathrm{min}$. Como é possível notar, os resultados de circularidade para as duas primeiras velocidades de avaço se mantiveram bastante próximos para as técnicas de lubri-refrigeração de MQL e CAMQL, 3,96 $\mu \mathrm{m}$ e 3,92 $\mu \mathrm{m} ; 4,32 \mu \mathrm{m}$ e 4,28 $\mu \mathrm{m}$, respectivamente. Já para a condição mais severa analisada, a velocidade de avanço de $0,75 \mathrm{~mm} / \mathrm{min}$ os resultados de circularidade destas duas técnicas mencionadas apresentam uma maior variação devido à maior quantidade de calor presente na técnica de MQL. De acordo com ALVES [13] durante a retificação acontece o consequente empastamento do rebolo, a incrustação de cavacos na porosidade da ferramenta aumenta e este crescimento acaba ligando o material empastado com os grãos adjacentes, e este fenômeno se repete até que o grão esteja desgastado e se desprenda ou até que as forças de retificação sejam suficientes para a remoção do grão e do material aderido a ele. Conforme se tem um processo mais severo, caracterizado por uma maior velocidade de avanço do rebolo, as forças de usinagem se tornam maiores, fazendo com que as incrustações citadas possam ser removidas com mais facilidade, ou seja, a dificuldade dessa remoção acontece em situações de usinagem mais branda, como é o caso das duas primeiras velocidades de avanço, o que explica o comportamento dos resultados para estas situações.

Para a técnica de lubri-refrigeração convencional, os resultados obtidos para a circularidade foram 2,23 $\mu \mathrm{m}$ para a velocidade de avanço de $0,25 \mathrm{~mm} / \mathrm{min}, 3,34 \mu \mathrm{m}$ para a velocidade de avanço de $0,50 \mathrm{~mm} / \mathrm{min}$ e $3,83 \mu \mathrm{m}$ para a velocidade de avanço de $0,75 \mathrm{~mm} / \mathrm{min}$. Como é possível notar estes foram os menores resultados obtidos para o parâmetro de forma e isso acontece pois a lubri-refrigeração convencional tem uma 
maior capacidade de lubrificação e a mesma é capaz de manter a região de corte livre de detritos, o que ocasiona em uma maior precisão dimensional.

\subsection{Desgaste diametral do rebolo}

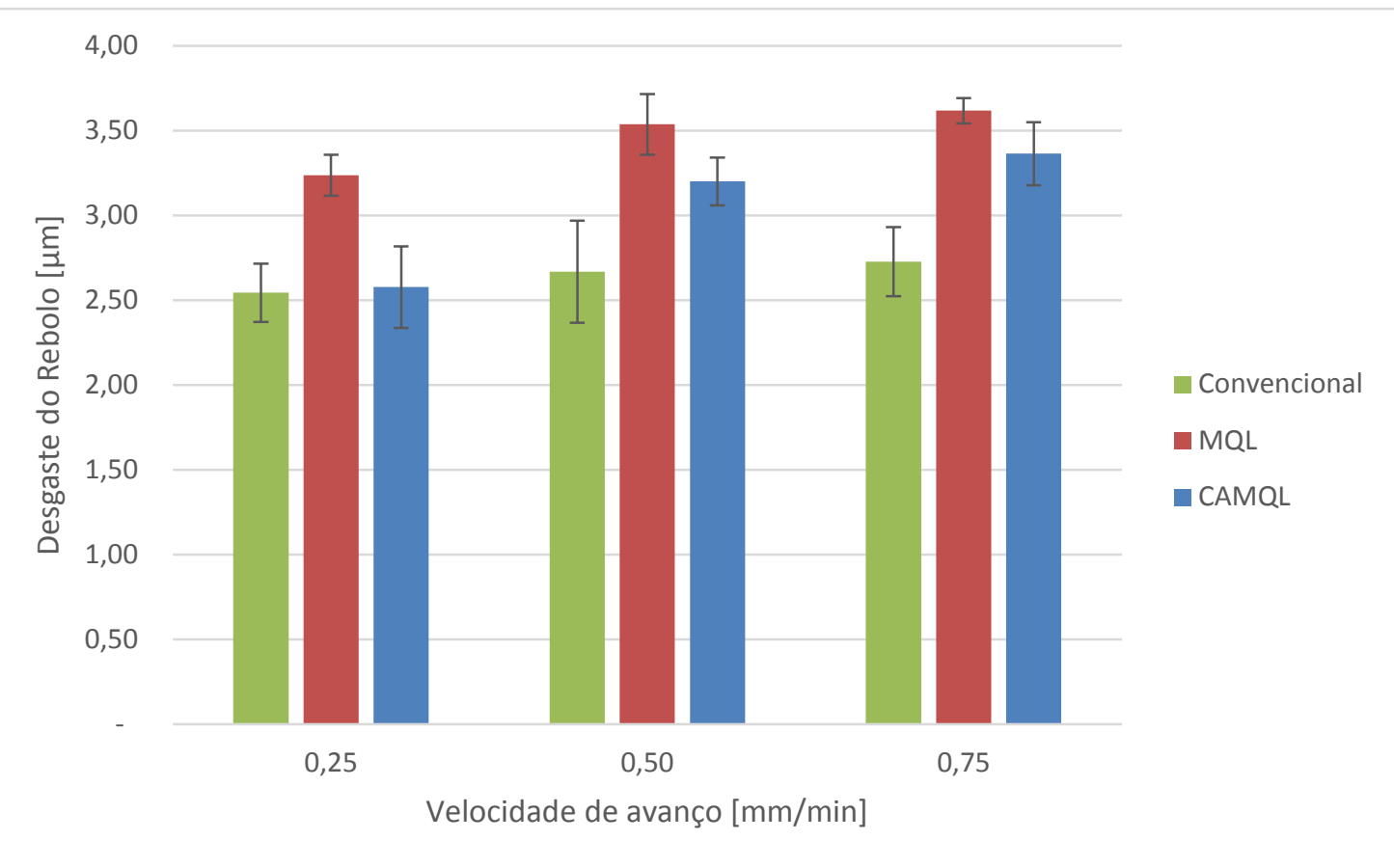

Figura 7: Dados obtidos de desgaste diametral do rebolo em função da velocidade de avanço.

Os resultados para o desgaste da técnica de lubri-refrigeração convencional foram de 2,54 $\mu \mathrm{m}, 2,67$ $\mu \mathrm{m}$ e $2,73 \mu \mathrm{m}$ para as velocidades de avanço $0,25 \mathrm{~mm} / \mathrm{min}, 0,50 \mathrm{~mm} / \mathrm{min}$ e $0,75 \mathrm{~mm} / \mathrm{min}$.

Para o método de mínima quantidade de lubrificação ou MQL os resultados do desgaste diametral do rebolo foram os mais elevados obtidos, sendo de 3,24 $\mu \mathrm{m}$ para a velocidade de avanço de $0,25 \mathrm{~mm} / \mathrm{min}, 3,54 \mu \mathrm{m}$ para a velocidade de avanço de $0,50 \mathrm{~mm} / \mathrm{min}$ e $3,62 \mu \mathrm{m}$ para a velocidade de avanço de $0,75 \mathrm{~mm} / \mathrm{min}$. Por fim temos os valores intermediários de desgaste diametral do rebolo da técnica de CAMQL ou mínima quantidade de lubrificação com ar comprimido frio, os quais foram 2,58 $\mu \mathrm{m}, 3,20 \mu \mathrm{m}$ e 3,36 $\mu \mathrm{m}$ para as respectivas velocidades de avanço.

Observando estes valores é possível observar que o valor do desgaste diametral do rebolo para a técnica de CAMQL ficou bastante próximo dos resultados para o método convencional, sendo respectivamente $2,58 \mu \mathrm{m}$ e $2,54 \mu \mathrm{m}$, para a velocidade de avanço de $0,25 \mathrm{~mm} / \mathrm{min}$. Isso é justificado pela combinação de dois fatores distintos, os quais são a condição de corte mais branda com o baixo acúmulo de calor propiciada pelo método de CAMQL, pois o desgaste do rebolo pode acontecer pela degradação térmica de uma alta quantidade de calor presente na interface de corte, e por não haver essa concentração de calor na região de corte, graças às razões expostas acima as duas técnicas apresentaram resultados bastante próximos.

O resultado do desgaste diametral do rebolo da técnica de CAMQL ficou próximo da técnica de MQL para a condição mais severa de usinagem, a velocidade de avanço de $0,75 \mathrm{~mm} / \mathrm{min}$, com valores de desgaste de 3,36 $\mu \mathrm{m}$ e 3,62 $\mu \mathrm{m}$ respectivamente, o que pode ser explicado pela condição mais severa de corte, já que há uma maior geração de calor e consequentemente um maior acúmulo de energia na região de corte, o que leva à degradação térmica dos grãos e ligante, prejudicando a força de adesão entre estes dois elementos e levando à um maior desgaste. 


\subsection{Potência de corte}

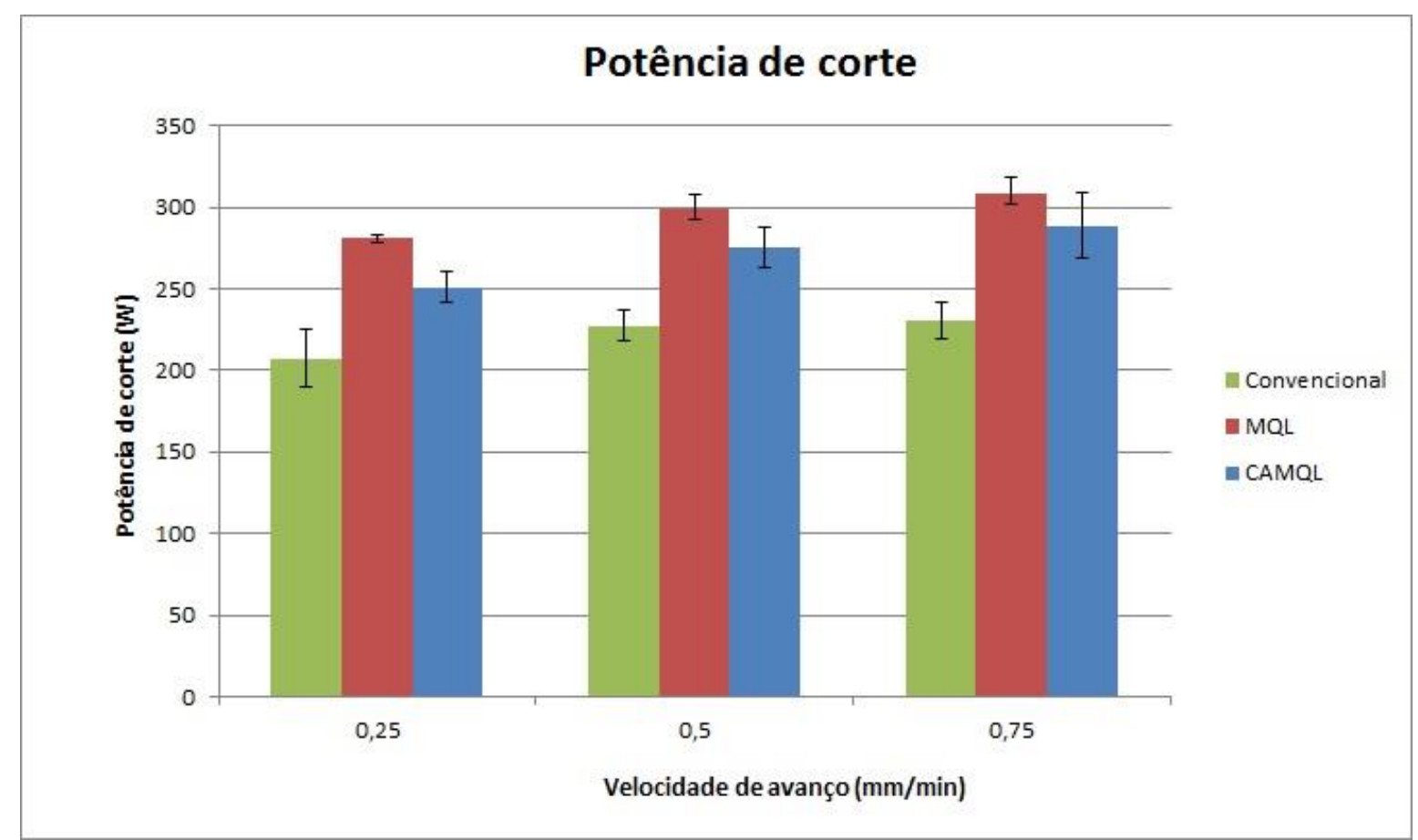

Figura 8: Dados obtidos de potência de corte em função da velocidade de avanço.

Como é possível notar, o gráfico de potência de corte apresenta a mesma tendência que todos os outros anteriores, o aumento dos valores com o aumento da velocidade de avanço. $\mathrm{O}$ aumento da velocidade de avanço aumenta a quantidade de material retirado por passe, e quando isso acontece há um aumento da área de desgaste plano e a profundidade de corte de cada um dos grãos, por isso uma maior energia é necessária para a remoção de material. [14]

Nota-se que os valores de potência para a técnica de lubri-refrigeração convencional obteve os menores valores, 207,33 W para a velocidade de avanço de $0,25 \mathrm{~mm} / \mathrm{min}, 227,67 \mathrm{~W}$ para a velocidade de avanço de $0,50 \mathrm{~mm} / \mathrm{min}$ e $230,71 \mathrm{~W}$ para a velocidade de avanço de $0,75 \mathrm{~mm} / \mathrm{min}$. Este método fornece a melhor lubrificação de todos os métodos estudados, também fornece uma boa refrigeração, o que permite que o corte seja realizado de forma mais fácil, com uma necessidade de menos energia para remover a mesma quantidade de material, também se deve levar em consideração que a geração de calor permanece sob controle com a aplicação deste método, e a combinação destes fatores leva a uma menor potência requerida durante o corte.

Já o método de mínima quantidade de lubrificação, ou MQL fornece os mais altos resultados para a potência, $281,74 \mathrm{~W}, 298,96 \mathrm{~W}$ e $308,40 \mathrm{~W}$ para as velocidades de avanço de $0,25 \mathrm{~mm} / \mathrm{min}, 0,50 \mathrm{~mm} / \mathrm{min}$ e $0,75 \mathrm{~mm} / \mathrm{min}$, respectivamente. Isso acontece, pois a lubrificação fornecida por esta técnica não é suficiente para que o desempenho do corte seja obtido satisfatoriamente. A falha de lubrificação faz com que seja mais difícil para os grãos abrasivos removerem o material da peça e assim a potência de corte atinge valores elevados. Outro ponto importante é a alta geração de calor que esta técnica de lubri-refrigeração não pode controlar, e este fator favorece o desgaste plano dos grãos abrasivos o que aumenta a energia requerida para que ocorra a remoção de material.

Por fim, o método de mínima quantidade de lubrificação com ar comprimido resfriado ou CAMQL apresenta resultados melhores que a técnica de MQL, com valores de 251,09 W, 257,75 W e 288,62W para as respectivas velocidades de avanço $0,25 \mathrm{~mm} / \mathrm{min}$. $0,50 \mathrm{~mm} / \mathrm{min}$ e $0,75 \mathrm{~mm} / \mathrm{min}$. Em comparação com a técnica de MQL com ar em temperatura ambiente, o presente método é capaz de controlar melhor a quantidade de calor acumulada na região de corte, facilitando assim a remoção de material e consequentemente gerando menos energia. Porém esta técnica não é capaz de fornecer lubrificação suficiente para o corte, por isso seus valores de potência ficam mais elevados que o método convencional de lubri-refrigeração. 


\section{CONCLUSÕES}

Através dos resultados obtidos pelo presente trabalho, as conclusões obtidas serão listadas a seguir.

- A técnica de lubri-refrigeração convencional apresentou os melhores resultados para todos os parâmetros analisados, tudo isso graças à sua capacidade de lubrificação e de retirada dos detritos da região de corte;

- $\quad$ A técnica de lubri-refrigeração de mínima quantidade de lubrificação apresentou os piores resultados em todos os parâmetros já que a mesma não oferece refrigeração suficiente e também causa a formação de borra na superfície de corte do rebolo;

- $\quad$ A técnica de lubri-refrigeração de mínima quantidade de lubrificação combinada com ar comprimido resfriado apresenta resultados intermediários, pois é capaz de fornecer a refrigeração que a técnica de MQL não fornece, porém também apresenta a formação de borra o que deteriora os resultados e impede que ela seja melhor que a técnica convencional;

- Se o objetivo for a redução da quantidade de fluido de corte utilizado durante o processo de retificação, a técnica de CAMQL é indicada, pois apresenta melhores resultados quando comparada com a técnica de MQL, porém se apenas a qualidade do processo for levada em consideração, a técnica de lubri-refrigeração convencional deverá ser utilizada.

\section{AGRADECIMENTOS}

Os autores agradecem ao Departamento de Engenharia Mecânica da Faculdade de Engenharia da UNESP de Bauru - SP, à Coordenação de Aperfeiçoamento de Pessoal de Nível Superior - CAPES, à Fundação de Amparo à Pesquisa do Estado de São Paulo - FAPESP pelo apoio financeiro, à Nikkon Ferramentas de Corte Ltda., pertencente ao grupo Saint-Gobain, pelo doação do rebolo de CBN, à Quimatic Tapmatic Brasil Ltda. e à ITW Chemical Products Ltda. pela doação dos fluidos de corte e à Emuge-Franken Ferramentas de Precisão Ltda. pela doação do tubo de vórtice e a todos pela oportunidade de pesquisa .

\section{BIBLIOGRAFIA}

[1] BABIC, D., MURRAY, D. B., TORRANCE, A. A., "Mist jet cooling of grinding processes", Journal Machine Tools Manufacture, v. 45, n. 10, pp. 1171-1177, 2005.

[2] SANCHEZ, J. A., POMBO, I., ALBERTI, B., et al., "Machining evaluation of a hybrid MQL-CO2 grinding technology", Journal of Cleaner Production, v. 18, n. 18, pp. 1840-1849, Jul. 2010.

[3] BELENTANI, R.M., JÚNIOR, H.F., CANARIM, R.C., et al., "Utilization of minimum quantity lubrication (MQL) with water in CBN grinding of steel", Material Research, v. 17, n. 1, pp. 88-96, 2014.

[4] OLIVEIRA, J.F.G., SILVA, E.J., COELHO, R.T., et al., "Dry grinding process with workpiece precooling" CIRP Annals - Manufacturing Technology, v. 64, pp. 329-332, 2015.

[5] DEBNATH, S., REDDY, M.M., YI, Q.S., "Environmental friendly cutting fluids and cooling techniques in machining: A review" Journal of Cleaner Production, v. 83, pp. 33-47, Ago. 2014.

[6] CHOI, H.Z., LEE, S.W., JEONG, H.D., "A comparison of the cooling effects of compressed cold air and coolant for cylindrical grinding with a CBN wheel”, Journal of Material Processing Technology, v. 111, n. $1-3$, pp. 265-268, 2001.

[7] IRANI, R.A., BAUER, R.J., WARKENTIN, A., "A review of cutting fluid application in the grinding process" International Journal of Machine Tools \& Manufacture, v. 45, n. 15, pp. 1696-1705, 2005.

[8] HADAD, M.; SADEGHI, B., "Thermal analysis of minimum quantity lubrication-MQL grinding process", International Journal of Machine Tools \& Manufacture, vl. 63, pp. 1-15, 2012.

[9] PAUL, P.S., VARADARAJAN, A.S., GNANADURAI, R.R., "Study on the influence of fluid application parameters on tool vibration and cutting performance during turning of hardened steel", Engineering Scienci and Technology, an International Journal, v. 19, n. 1, pp. 241-253,Set. 2015.

[10] FREDJ, N.B., SIDHOM, H., BRAHAM, C., "Ground surface improvement of the austenitic stainless steel AISI 304 using cryogenic cooling” Surface \& Coatings Technology, v. 200, n. 16-17, pp. 4846-4860, Jun. 2006.

[11] MORGAN, M., BARCZAK, L., BATAKO, A., "Temperatures in fine grinding with minimum quantity lubrication (MQL)" International Journal of Advanced Manufacturing Technology, v. 60, n. 9-12, pp. 951958, 2012.

[12] SABERI, A., RAHIMI, A.R., PARSA, H., et al., "Improvement of surface grinding process performance 
of CK45 soft steel by minimum quantity lubrication (MQL) technique using compressed cold air jet from vortex tube", Journal of Cleaner Production, v. 131, pp. 728-738, Mai. 2016.

[13] ALVES, M. C. S., Influência dos métodos de lubri-refrigeração na retificação do aço ABNT 4340 usando rebolo de CBN, Tese de D.Sc, Universidade Estadual Paulista, Faculdade de Ciências,Bauru, SP, Brasil, 2007.

[14] PEREIRA, O., CATALÀ, P, RODRÍGUEZ, A., et al., "The Use of Hybrid CO2+MQL in Machining Operations" Procedia Engineering, v. 132, pp. 492-499, 2015.

\section{ORCID}

Kamira Miksza Fragoso José Claudio Lopes Hamilton José de Mello Alan Polato Francelin Luiz Eduardo de Angelo Sanchez Paulo Roberto de Aguiar Eduardo Carlos Bianchi https://orcid.org/0000-0002-4305-5402

https://orcid.org/0000-0001-5517-1016

https://orcid.org/0000-0002-7887-204X

https://orcid.org/0000-0003-1300-7474

https://orcid.org/0000-0002-6866-5055

https://orcid.org/0000-0002-9934-4465

https://orcid.org/0000-0003-2675-4276 